\title{
Visibility maps and spherical algorithms
}

\author{
Tony C Woo
}

\begin{abstract}
By the extraction of ideas from computer vision, geometrical design and complexity analysis, a structure called visibility emerges. The paper describes a way in which a 3D workpiece is mapped onto the unit sphere, and its visibility is determined. For applications, manufacturing machines are classified by their degrees of freedom into point, line and surface visible processes. Algorithms for optimal workpiece orientation are then formulated as simple intersections on the sphere.
\end{abstract}

Keywords: manufacturing, computational geometry, data structures, algorithms, surface normals, curvature, mappings

Convexity is a notion that facilitates geometrical computation. An entity is said to be convex if the line segment $p q$ that joins two arbitrary points $p$ and $q$ from a set lies entirely in the set. However, entities such as curves and surfaces are not always convex. The notion of visibility promises to extend beyond convexity. Take the art-gallery formulation ${ }^{1}$, for example. Given a nonconvex polygon that resembles the floor plan of an art gallery, one asks how many guards are needed such that the entire interior is visible to the guards. Intuition suggests that the lines of sight, or point-to-point visibility, partition a nonconvex polygon into convex (visible) regions, hence bringing convexity to an entity that was nonconvex. This paper takes analytically complex entities, and maps them onto a unit sphere into analytically simple entities called visibility maps that turn out to be convex. Problems that are combinatorially complex are then addressed by a set of simple spherical algorithms that invoke the intersection between the visibility map and a point, a great circle or a spherical rectangle; these are also convex.

The establishment of a line of sight between a point on a workpiece and a point on an effector can be quite

Department of Industrial and Operations Engineering, University of Michigan, Ann Arbor, MI 48109-2117, USA

Paper received: 10 November 1992. Revised: 26 April 1993 useful in manufacturing. In numerical-control (NC) machining, assembly with robots, or inspection by coordinate-measurement machines (CMM), the computation for a tool path often relies on an intersection-free line of sight. Though the geometries of the effector of the manufacturing processes differ, the question about visibility in its most basic form can be stated as follows.

Basic enquiry about visibility: Given the geometries of the workpiece and the effector, find a number of orientations of the workpiece such that all the points of interest on the workpiece are visible to the effector.

That a minimum of orientations is crucial to productivity in manufacturing is evident. In an automotive application of the laser inspection of cylinder heads, the scanning time is approximately $20 \mathrm{~min}$ at $70 \mathrm{point} / \mathrm{line} / \mathrm{s}$. The setup time for fixturing and calibrating the workpiece is about $6 \mathrm{~h}$. Since a reorientation requires the dismounting and setting up of the workpiece, the total number of orientations (to achieve complete visibility) should be minimized.

To be sure, there are manufacturing operations in which only a single setup is affordable or permitted. The use of the stereolithography apparatus (SLA) in layered manufacturing is one such instance. (In layered manufacturing, a facsimile of a 3D computer representation is fabricated layer by layer, stacking along the $z$ axis. The material, which is a powder or a liquid, is selectively solidified by sintering or photocuring in the $x y$ plane. For sheet material, a contour in the $x y$ plane is created, and unwanted portions are discarded. However, regardless of the process, there is only one setup, and hence single implied orientation is permitted.) The choice of an orientation in layered manufacturing can make a difference. In an experiment in which the housing of a mobile phone was fabricated, two orientations showed a difference in fabrication time. In the orientation in which the antenna pointed upwards, it took over $22 \mathrm{~h}$ to fabricate the housing on an SLA 250. In the orientation 
in which the antenna pointed sideways, the fabrication time was less than $10 \mathrm{~h}$.

An intellectual curiosity emerges. If layered manufacturing is viewed as a physical realization of a triple integral, why should the order of integration matter? A layer or a crosssection $S$ can be thought of as a double integral:

$$
S=\iint_{c} \mathrm{~d} x \mathrm{~d} y
$$

where $c$ is a closed curve in the $x y$ plane. The integration of the layers $S$ along the $z$ direction yields a volume $V$ :

$$
V=\int S \mathrm{~d} z
$$

The same volume should result if, for instance, the crosssections are taken in the $y z$ plane or any other plane. However, an examination of the SLA manual reveals an explanation for the apparent paradox: the physical integration rates (the optical scanning rate in the $x y$ plane and the mechanical elevation rate in the $z$ direction) differ. Therefore, the physical constraint of a machine may lead to a preference about the order of integration, and hence the orientation of the workpiece.

Now, given that there exists asymmetry in time for a certain family of manufacturing processes, it is reasonable to expect that problem formulations for optimal workpiece orientations can be induced. However, what about manufacturing processes that do not exhibit asymmetry? In particular, time and ordering are important if the process is sequential. (NC machining, CMM probing, robotic assembly, and layered manufacturing with an SLA are all sequential, in the sense that physical realization is achieved point by point, layer by layer, or component by component.) Stamping, die sinking, metal casting and plastic mould injection, to name but a few, may be viewed as nonsequential or parallel in the sense that the entire workpiece is effected in one 'fell swoop'. For such manufacturing processes, does the same basic enquiry about optimal workpiece orientation still hold? The answer turns out to be yes. Consider the stamping operation as an example. The direction of travel for the die implies an orientation of the workpiece. The demoulding process is another: the parting plane in a 2-piece mould implies a direction for demoulding, and this is the same for decasting. This single degree of freedom dictates the visibility of the punch or the removability of the workpiece from the cast or the mould. Intuitively, there appears to be an inverse relation between the degrees of freedom of the mechanism of a machine and its visibility.

This paper introduces a hierarchy that classifies the various manufacturing processes by their visibilities. The geometries are then mapped onto a unit sphere offering a data structure called a visibility map. Algorithms for determining the optimal partitioning on the sphere are then developed, leading to answers to the basic enquiry about visibility.

\section{VISIBILITY HIERARCHY OF MANUFACTURING PROCESSES}

Visibility is meaningful when a pair consisting of a source and a target is considered. Geometrically, the target (or the workpiece) can be a point, a line segment, a curve segment, a planar polygon, or a surface patch. Similarly, the source (or the effector) can be modelled hierarchically by its topological dimensionalities: 0D points, 1D lines and curves, and 2D planes and surfaces, giving rise to a hierarchy, a summary of which is given in Figure 1. The geometries of the source are discussed in turn below.

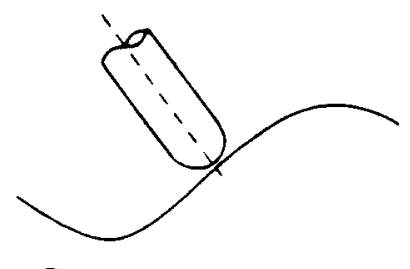

a

b
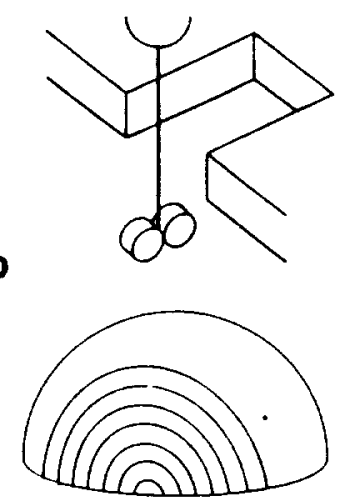

C
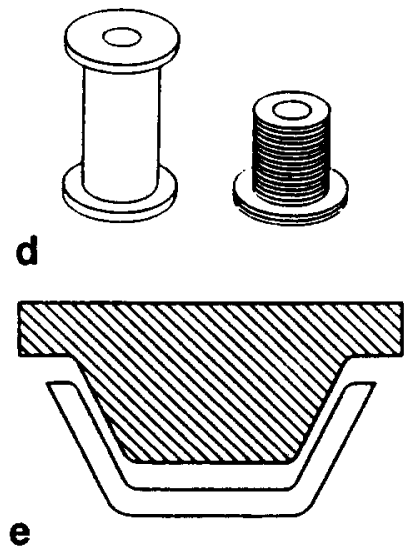

Figure 1 Visibility hierarchy; (a) point visibility, (b) line visibility, (c) curve visibility, (d) plane visibility, (e) surface visibility

[(a) numerically controlled machining, (b) wire-cutting electricdischarge machining, (d) stereolithography apparatus, (e) mould and die design.] 
The probe of a CMM is spherical; the contact that it makes with a workpiece under inspection is often assumed to be a point on the sphere. In NC machining, because of the small stepping distance now made available by the machine-tool makers (of $10^{-5}-10^{-6} \mathrm{~m}$ ), the material-removal process can also be considered as a point process. $\mathrm{NC}$ and $\mathrm{CMM}$ are thus said to be processes that require point visibility (which is short for point-to-point visibility).

For wire-cutting electric-discharge machining (EDM), the effector is a line. Because of the small radius of the cutting wire, fine features can be created on the workpiece. Also, because of the process of discharging, there must be line visibility between the effector and the workpiece such that all the points in a segment of the cutting wire are visible to the corresponding points in a line segment on the workpiece. A noncontact variation of the line-visibility process is laser scanning for inspection. Diffracting a point source by a cylindrical lens into a line source, the laser casts stripes of lines and curves onto the surface of a workpiece, giving rise to curve visibility.

In layered manufacturing, there must be plane visibility for an entire layer. The energy source of a layeredmanufacturing device can be conceptualized as a planar source, giving rise to a point-by-point correspondence between the source and the target. In the medical application of computer tomography, another noncontact variation, the slicing of the target can also be said to exhibit planar visibility.

The simplest example of surface visibility is probably sheet-metal stamping, in which the points on the sheet metal to be formed (the target) must maintain visibility with the corresponding points on the die (the source). Die-sinking EDM exhibits a similar characteristic of surface visibility. Similarly, die casting and plastics mould injection also demand surface visibility.

\section{Degrees of freedom and number of setups}

Recall that the amount of sequentialism varies from process to process. From Figure 1, it can be noted that, as the hierarchy traverses from surface to point visibility, the degrees of freedom (DOF) of the corresponding machines increases. While a sheet-metal stamping machine (possessing surface visibility) has only one DOF, an SLA (with planar visibility) has three DOF. Moving along the hierarchy, an $\mathrm{NC}$ milling machine (with point visibility) has up to five DOF.

Because of the way in which the hierarchy is established, the greater the number of degrees of freedom that a machine has, the simpler its unit work is. The unit work for an NC milling machine is to remove a small amount of material modelled as a point. In contrast, the unit work for a 1-DOF machine is the creation of a multiple number of surfaces in one motion. To enable the unit work to be done, the workpiece has to be set up properly. It also may be interesting to examine the tradeoff between the number of setups and the degrees of freedom afforded by the machine.

Consider a 3-axis NC milling machine and a 5-axis NC milling machine. Both are point-visible processes, with different degrees of freedom. Suppose, to avoid excessive gouging, a ball endmill is used in both NC machines. As shown in Figure 2, because of the fourth and the fifth axes which pivot the tool, a workpiece can be machined on a 5 -axis machine with a smaller number of dismountings, recalibrations and startups. Given that there is a choice of the machine to be used, it is reasonable to choose the one that offers fewer setups. This is a
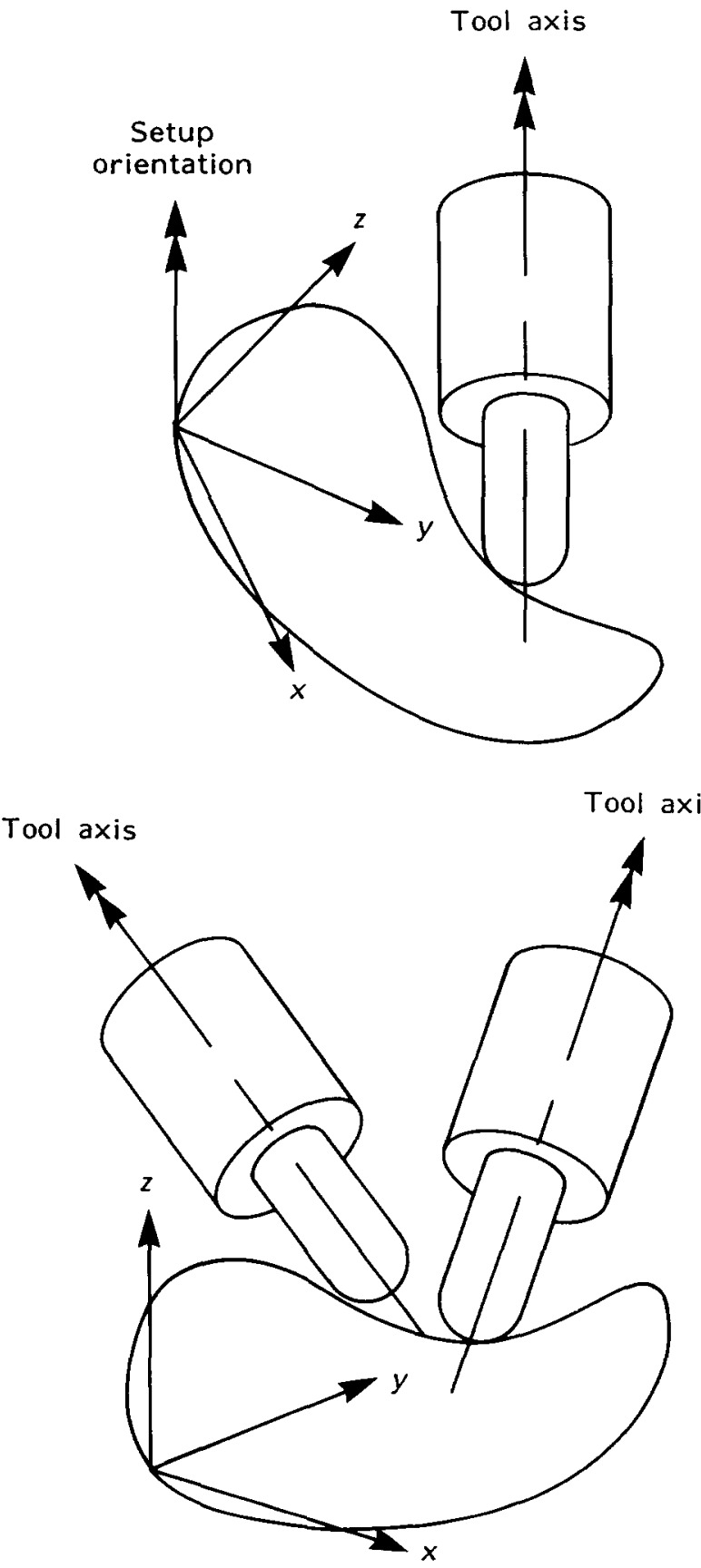

Figure 2 3-axis machining compared with 5-axis machining 
machine-selection problem that involves scheduling. However, given a fixed type of machine, say a 3-axis NC milling machine, it is also reasonable to ask whether the number of setups can be minimized. This leads to the following instance of the basic enquiry about visibility.

Enquiry about visibility with a high number of DOF: Given the geometries of the workpiece and the kinematics of the machine, find the minimum number of setups which require a reorientation of the workpiece.

\section{Partial visibility and auxiliaries}

A variation of the basic enquiry arises in manufacturing processes with a low number of degrees of freedom. Consider a surface-visible process such as moulding or casting. Suppose that the workpiece shown in Figure $3 a$ had been moulded. To remove the workpiece, the choice of the parting plane is crucial. The direction that is orthogonal to the parting plane yields the direction for the lines of sight, giving rise to the single degree of freedom in the demoulding process. Now, the limitations of a single parting plane (a 1-DOF surface-visible process) should be noted here. Regardless of the direction of 'illumination', there can always be 'shadows' and hence partial visibility in a cavity, as shown in Figure $3 b$.

Indeed, analogously to the number of setups for a process with a high number of DOF, there can be multiple pieces in the mould or die for a process with a low number of DOF. In place of the multiple pieces, auxiliaries (called 'cores' or 'pins') are used in practice. Figure 4 shows three kinds of auxiliaries in a 2-piece mould. To reduce the number of auxiliaries which incur expense, it is desirable to minimize them, leading to the second variation of the basic enquiry.

Enquiry about visibility with a low number of DOF: Given the geometries of the workpiece and the kinematics of the machine, find the minimum number of auxiliaries that compensate for partial visibility.

In the fourth section, the 'low' and 'high' numbers of DOF in these two enquiries are instantiated, and the two variations of the basic enquiry are once again generalized as intersections of spherical maps by a point, a great circle and a spherical rectangle. Before this is done, a discussion on the data structure of spherical maps is required.

\section{VISIBILITY MAP: A DATA STRUCTURE}

\section{Complete visibility}

Analogously to a hodograph ${ }^{2}$, which is a map of tangents, a Gauss map ( $\mathrm{G}$ map) is a map of normals on the unit sphere $^{3,4}$. The $G$ map has many applications. It provides cutting-tool orientations ${ }^{5}$ and surface offsets ${ }^{6,7}$ for NC machining, and it assists in computing the aspect graph ${ }^{8}$, and in shape reconstruction from imaging ${ }^{9}$ in computer vision. In the following discussion, given a surface $S$ in

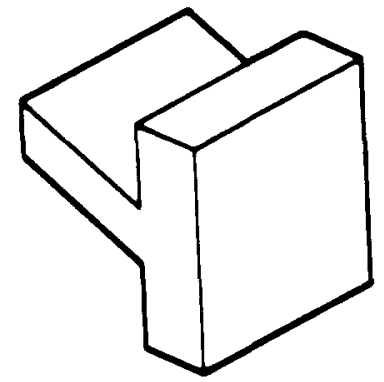

a

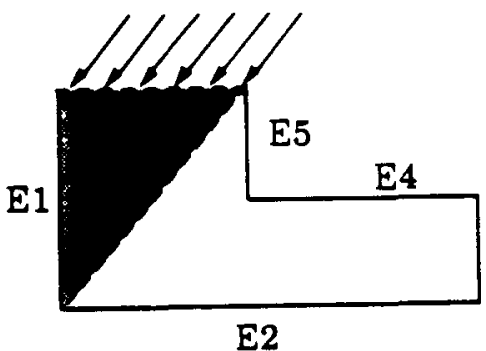

E3

b

Figure 3 Demoulding; (a) visibility, (b) partial visibility
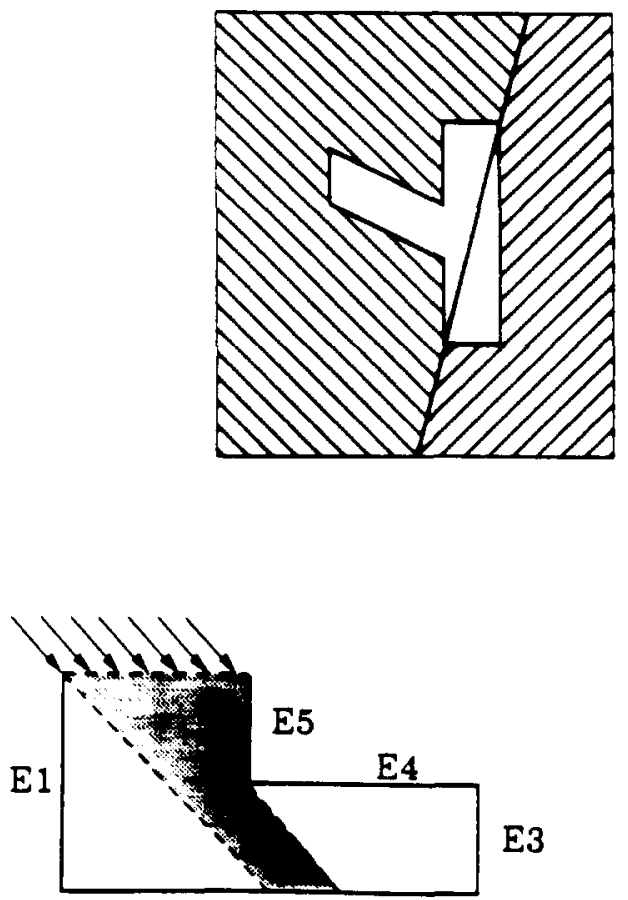

E2

E3

3 


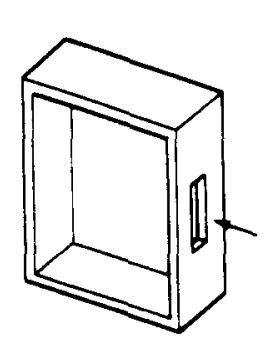

a

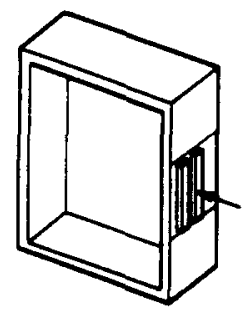

b

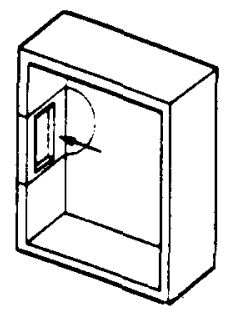

c
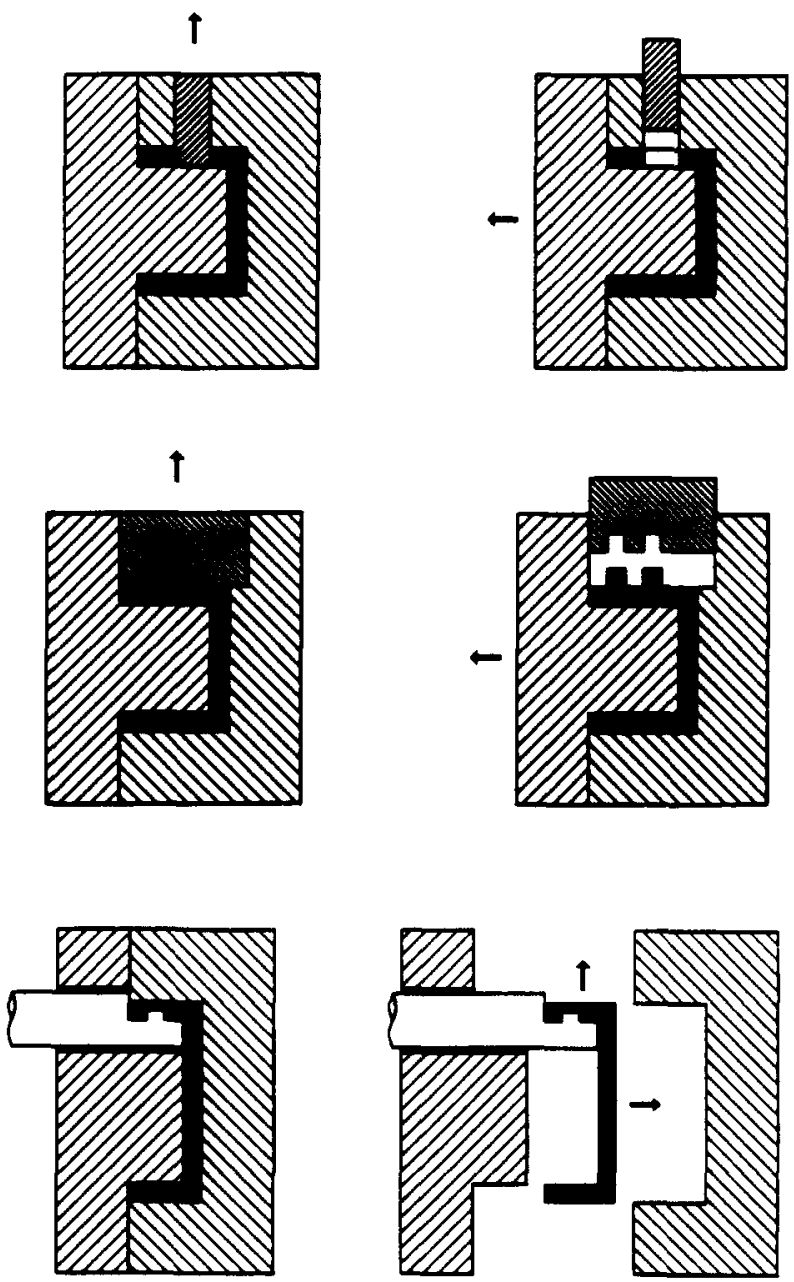

Figure 4 Auxiliaries in mould; (a) side core, (b) side cavity, (c) form pin

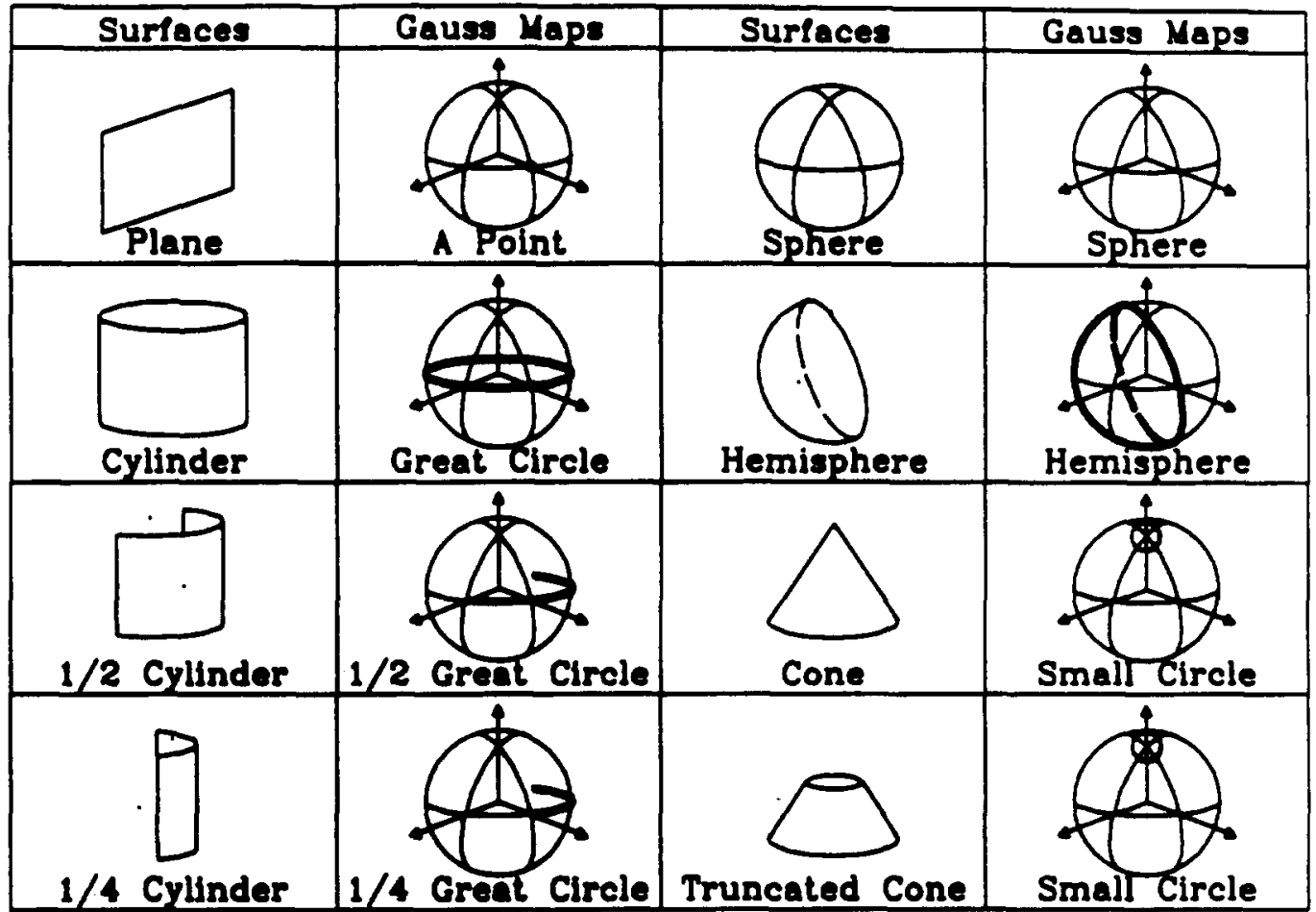

Figure 5 Examples of Gaussian maps 
a parametric form, it is assumed that its $G$ map exists. Figure 5 gives examples of $\mathrm{G}$ maps that are regions on the unit sphere. Note that each point in a $\mathrm{G}$ map denotes a direction from which a point in the surface is visible.

$A$ visibility map (or $V$ map) is an enhanced $G$ map in the following sense. Like a $G$ map, a $V$ map is a spherical region. However, unlike a $G$ map, any point in a $V$ map denotes a direction such that the entire surface $S$ is visible to its exterior. The idea of this powerful notion is simple. The normal at a point on a surface gives the direction in which the point is visible from infinity if the ray admits no intersection. However, the same point can be visible from many other directions, up to a hemisphere of directions bounded by the tangent plane at that point. This observation leads to a (rudimentary) procedure for computing a $\mathrm{V}$ map from a given $\mathrm{G}$ map. (The variable names are now joined by an underscore, to distinguish them from the procedure names.)

Procedure V map (G_map)

(0) $V_{-} M a p \leftarrow$ entire sphere

(1) For each point $p_{i}$ on $G$ map

$$
V_{-} \text {map } \leftarrow V_{-} M a p \cap \operatorname{hem} i\left(p_{i}\right)
$$

End V map

Figure 6 shows the V-map procedure in graphical form.

In Step 1, two calls are made: $\cap$ for computing the intersection of two sets on a sphere, and hemi $\left(p_{i}\right)$ for providing a hemisphere whose 'pole' is at point $p_{i}$. The following observations can be made about these two calls in relation to the efficiency of computing a $V_{-}$map. First, an intersection can be computed quickly if the two sets are convex. A hemisphere is a convex set. (This can be verified easily through central projection. The great circle bounding a hemisphere maps into a line in the plane of projection, and the hemisphere maps into a half plane.) Since the intersection of convex sets is always convex, the $V_{-}$map generated by successive intersections of hemispheres at $p_{i}$ must also be convex. Second, revisiting Step 1 of the V-map procedure, it is noted that the number of iterations is not specified. In particular, if a $G_{-}$map is

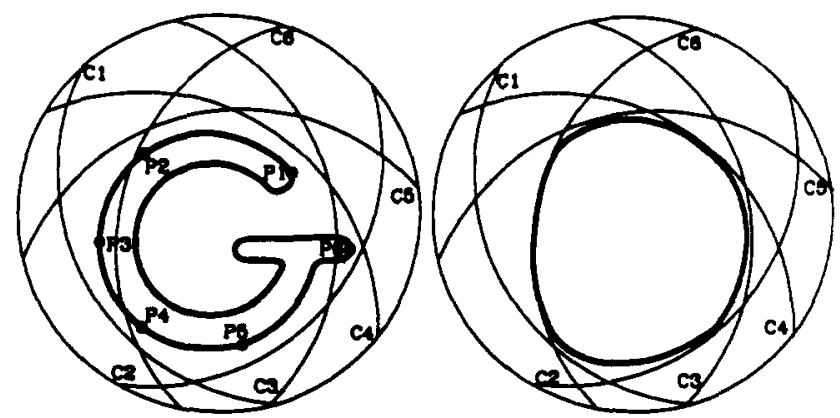

Figure 6 Construction of visibility maps to be sampled at $p_{i}$, should its entire interior be included? (Recall that a $G \_$map is not necessarily convex, unlike a $V \_m a p$. To ensure that a sample $p_{i}$ falls into a $G_{-}$map, it has to be tested by counting the even/odd number of intersections between a ray emanating from $p_{i}$ and the boundary of the $G_{-}$map, all adding to the time complexity.) Two steps are crucial to achieving savings in computation time. First, if only the boundary of a $G \_$map is sampled, then there is no need for ray testing. (This sufficiency condition is shown below.) Second, the boundary of a $G_{-}$map can be quite arbitrary, as it is, in general, nonconvex. If only the convex portion of the boundary of a $G$ map is sampled, there can be further savings. (The necessity is also established below.)

Suppose that $p_{i}$ is any point in the interior of a $G_{-}$map. Let $C$ be a circle passing through it. $C$ must make at least two intersections $q_{j}$ and $q_{k}$ with the boundary of the $G_{-}$map that lie on either side of $p_{i}$. Construct three hemispheres whose poles are at the three points $p_{i}, q_{j}$ and $q_{k}$ on $C$. If hem $i\left(q_{j}\right)$ intersects hemi $\left(q_{k}\right)$, and they must, unless $q_{j}$ and $q_{k}$ are exactly $180^{\circ}$ apart, it is seen that hem $i\left(p_{i}\right)$ is redundant in its contribution to the $V_{-}$map. This establishes the sufficiency of sampling the boundary of a $G_{-} m a p$. Now, the necessity of sampling the convex portion of the boundary follows a similar reasoning. Let $p_{i}$ be any point on the nonconvex portion of the boundary, and the other two points $q_{j}$ and $q_{k}$ be on the convex portion. (The three points must coexist on any great circle $c$ passing through $p_{i}$ ). It is seen that, again, hem $i\left(p_{i}\right)$ is redundant, hence establishing the necessity criterion.

Enjoying convexity, the V-map procedure can be implemented in time $\mathrm{O}(n \log n)$, where $n$ is the number of samples on the spherical convex hull of a $G_{-}$map. (For further details on the intersection of hemispheres, the construction of a spherical convex hull, and the determination of whether a given set of points lies in a hemisphere, the reader is referred to Reference 10.) A $V \_$map can now be computed quickly. Further, its representation can be compacted. An incircle, a spherical circle inscribed in a $V_{-}$map, gives a conservative approximation, and is much more compact in its representation. However, unlike circumscription, inscription is very difficult, because of the lack of 'extreme points'. In Reference 11, a 2-step procedure for finding the incircle of a $V_{-}$map is described. The first step is to take the farthest point Voronoi diagram of a $G_{-}$map to obtain the circumcircle for a $G_{-}$map. Next, it is shown that its $180^{\circ}$ complement yields the desired incircle of a $V \_$map. In Reference 11 , implementations of the G-map procedure and the V-map procedure are also given.

\section{Partial visibility}

A $V \_$map obtained from a $G \_$map which in turn is computed from a given surface $S$ has the following property. Any point in a $V_{-}$map yields a direction such 
that the entire $S$ is potentially visible to the exterior. For $n$ surfaces to be visible, their corresponding $V$ _maps must overlap. Sometimes, the overlap is empty. As shown in Figure 7, the planar surfaces yield single points as their G_maps; their $V_{-}$maps are hemispheres, the intersections of which are empty. Indeed, there is not a single direction from which the interior of the entire cavity is visible from the exterior.

Given that the visibility is partial, there will be 'shaded' regions for a given direction of illumination. (In the context of manufacturing, the shaded regions correspond to the auxiliaries, and the direction of illumination to the single degree of freedom, as in demoulding.) Figure 8 shows two configurations. It can be seen that the geometry and the topology of the auxiliaries differ in these two instances. In the viewing direction of Figure $8 a$, the geometry of the auxiliary changes, but its topology remains invariant, as the viewing direction is changed slightly. The transition from Figure $8 a$ to Figure $8 b$ is termed the critical viewing direction (CVD). Once a CVD has been determined, the augmented visibility maps ( $A_{-} V_{-}$maps) can be constructed, in which hemispheres corresponding to the (planar) surfaces are intersected. (Note that, unlike a $V_{-}$map, an $A_{-} V_{-}$map is valid only with respect to a CVD.) The (shaded) auxiliaries can be computed by taking the regularized set difference between the cavity in question and the (unshaded) visibility polyhedron ${ }^{12}$. Because the visibility polyhedron is a proper subset of the cavity, an $\mathrm{O}(n \log n)$ time algorithm ${ }^{13}$ can be used in computing for their intersection.

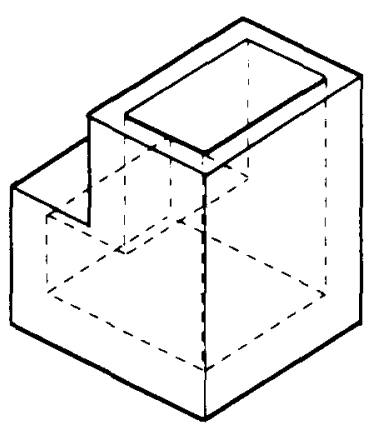

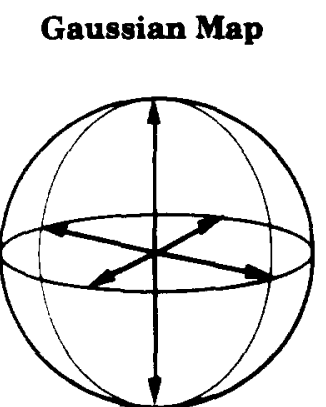

Figure 7 Empty visibility map
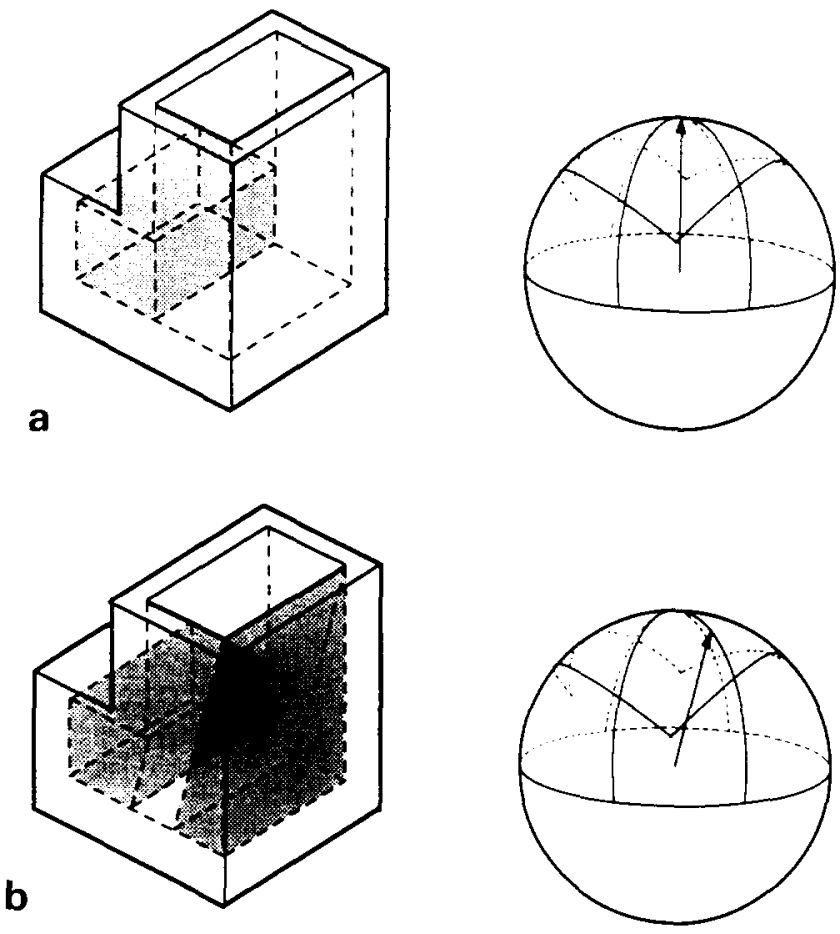

Figure 8 Augmented visibility maps

\section{SPHERICAL ALGORITHM}

The workpiece geometry having been transformed onto the unit sphere as $V_{-}$maps, it is time to see how the mechanisms of the machines are related to the geometry of the workpiece. The basic idea is as follows. Suppose that the degrees of freedom of the mechanism of a machine can be represented, in terms of visibility, on a unit sphere. Then, given the $V_{-}$maps which are also on a unit sphere, the problem of setting up a workpiece on a machine becomes that of rotating the sphere with the $V \_$maps against that with the machine, according to some criteria. A criterion is maximal intersection. Rotate one of the two nested unit spheres such that their maps intersect maximally. (This corresponds to a setup in which the greatest number of workpiece surfaces are visible to the machine.) Delete those intersected $V_{-}$maps, and continue until all the $V_{-}$maps of interest are deleted. Relating the visibility hierarchy to the degrees of freedom is now appropriate. A summary is given in Figure 9. The entities on the sphere, a point, a segment of a great circle, and a spherical rectangle, denote the varying DOF of the machines. For clarity, the $V_{\text {maps }}$ representing the workpiece geometry are omitted from the figure.

First, consider a 3-axis NC milling machine. Let the three DOF of the machine be denoted by $x, y, z$, meaning that they are the translations in the $x, y$ and $z$ directions. The tool axis of such a machine can be represented by a point $p$ on the unit sphere. In other words, the visibility of the 3-DOF NC machine is in the direction that is opposite to the vector $p$ represented as a point on the unit sphere. To machine a workpiece, the $V_{-}$maps of the 


\section{Point Visibility Visibility \\ Surface \\ Visibility}

1 DOF

2 DOF

4 DOF
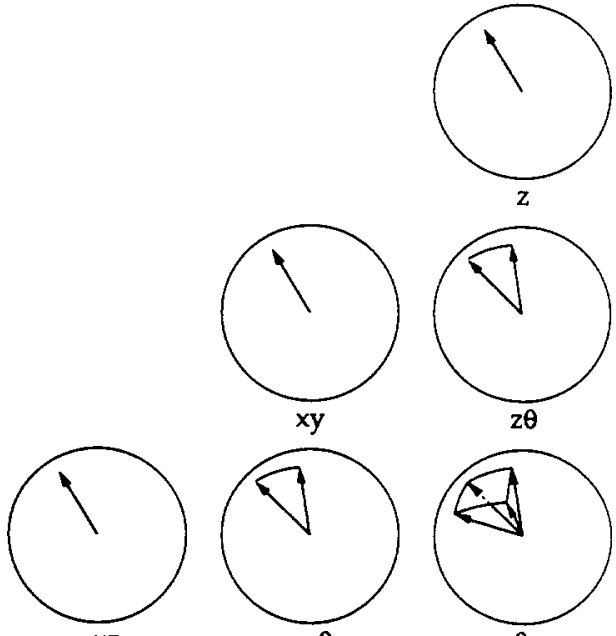

$3 \mathrm{DO}$
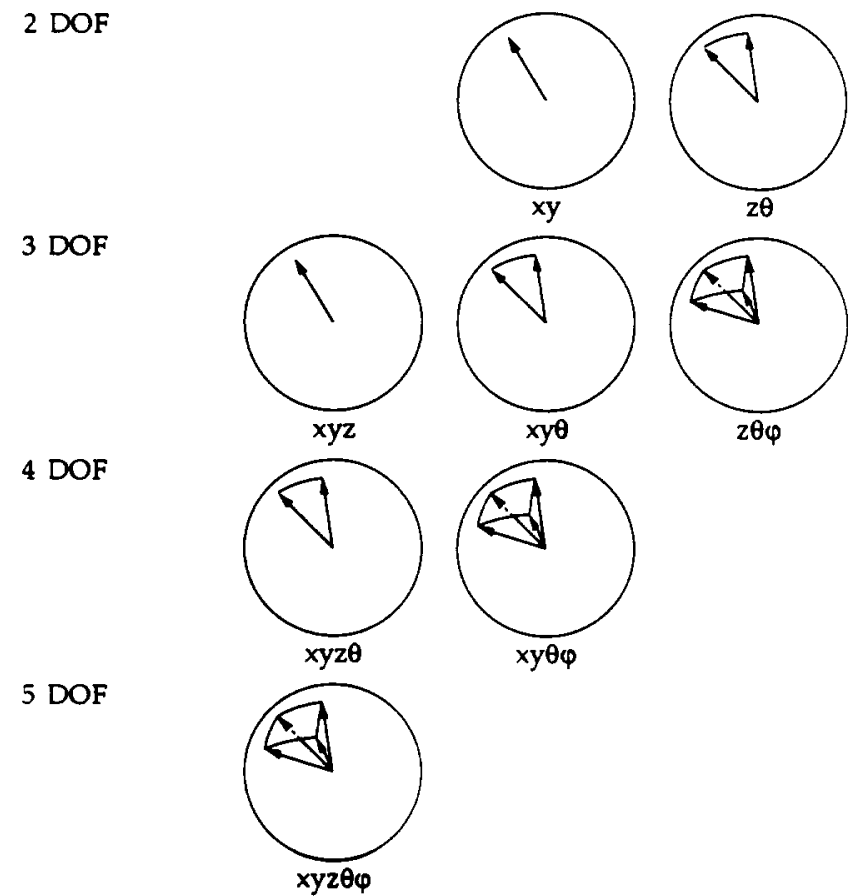

Figure 9 Spherical algorithms

workpiece must be oriented by rotating the maps and keeping the point $p$ fixed in such a way that the $V \_$maps contain $p$. The rotation of the $V_{-}$maps therefore corresponds to a setup. It is obvious that the point $p$ may not lie in all the maps. Dismounting the workpiece, reorienting, and reclamping the workpiece (another setup) permits other maps to contain the point $p$, and hence the visibility and machining of other portions of the workpiece. This corresponds to three DOF and point visibility, as shown in Figure 9.

Next, consider a 4-axis NC milling machine with a rotary table. Let the fourth axis be denoted by $\theta$. Figure 10 shows an example of such a 4-axis NC machine. Intuitively, the additional axis offers greater visibility than a 3-axis machine. Indeed, the additional degree of freedom from the rotary table can be represented by a great circle $C$ on the unit sphere, if the table rotates through $360^{\circ}$. (In Figure 9, a segment of a great circle is shown.) To ensure the visibility of the workpiece on a four-DOF point-visible machine, the $V_{-}$maps must be rotated in such a way that they are maximally intersected by $C$.

A 5-axis NC machine has an additional degree of freedom, denoted by $\phi$. This rotary DOF is again a segment of another great circle $C^{\prime}$ on the unit sphere. (It is customary that the fifth axis does not rotate through $360^{\circ}$ to prevent the fixtures and the table from tool collision and damage.) The combination of the fourth and the fifth axes forms a band, or just a spherical rectangle $R$ on the sphere. To ensure visibility, the maps must again be rotated such that they are intersected by $R$.

The enquiries about visibility for machines with low and high numbers of degrees of freedom posed in the second section can now be succinctly stated in terms of the relation between the maps (which represent the workpiece geometry and the effector geometry) and three entities: a point, a great circle, and a spherical rectangle (which represent the increasing number of degrees of freedom in the mechanism of the machine), all on a unit sphere.

Visibility problem: Given the geometries of the workpiece and the effector, and the kinematics of the machine, find an orientation of the maps such that they are intersected by a point, a great circle or a spherical rectangle.

Returning to Figure 9, it can be seen that the visibility problem covers all the manufacturing processes that possess point visibility, line visibility, or surface visibility. Take the 1-DOF surface-visible process as an example, one instance of which is stamping. The relative orientation of the punch and the sheet metal on the die must be such that the direction of travel offers visibility to all the points on the workpiece, so as to result in the desired shape in the sheet metal bound on the die. The removal of a casting from a 2-piece cast is another example. The parting plane must be such that the casting can be removed in the direction normal to the parting plane. The direction of the punch and the direction of the casting can both be represented by a point on the unit sphere; the single DOF is denoted by $z$, without loss of generality.

Finally, the line-visible processes in the middle column of Figure 9 show the formulation of the visibility problem. Consider a 2-DOF line-visible process such as the wire-cutting EDM. Let the direction of the cutting wire be in the $z$ axis, represented by a point $p$ on the unit sphere. There are two degrees of freedom $x$ and $y$ for the cutting wire to travel. As shown in Figure 11, such a configuration of a wire-cutting EDM can create planar surfaces whose normals are in the $x$ or $y$ direction. Consider the surface facing in the $x$ direction. Its G_map is a point $g_{1}$ on the unit sphere. Now, the same surface can be cut in many directions, some of which are shown by the crosshatching on the surface in Figure 11. In fact, the cutting wire can assume any of the directions for all the lines lying in the plane facing in the $x$ direction; they form a $V_{-}$map which is a great circle $V_{1}$ on the unit sphere. Similarly, all the possible cutting directions for the surface facing in the $y$ direction yield a $V_{-}$map of another great circle $V_{2}$. To cut both surfaces, their $V_{-}$maps must intersect, and they do, at the 'north pole'. Hence, the cutting wire must be oriented along the $z$ axis, coinciding with the pole. No other orientation would 

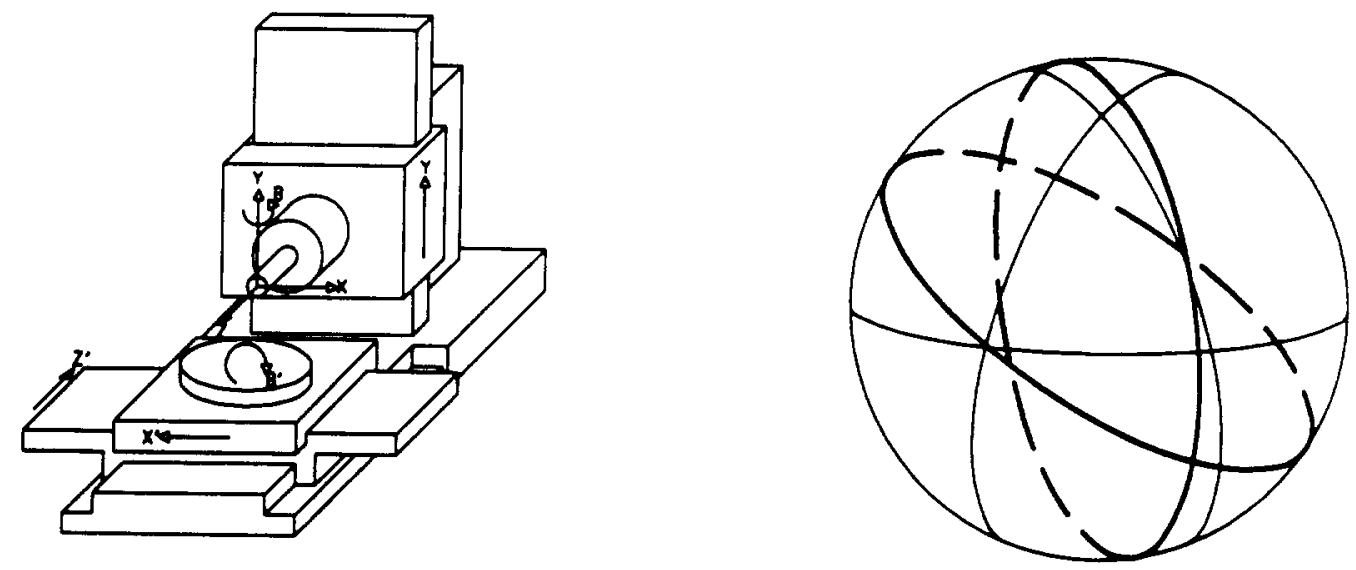

a
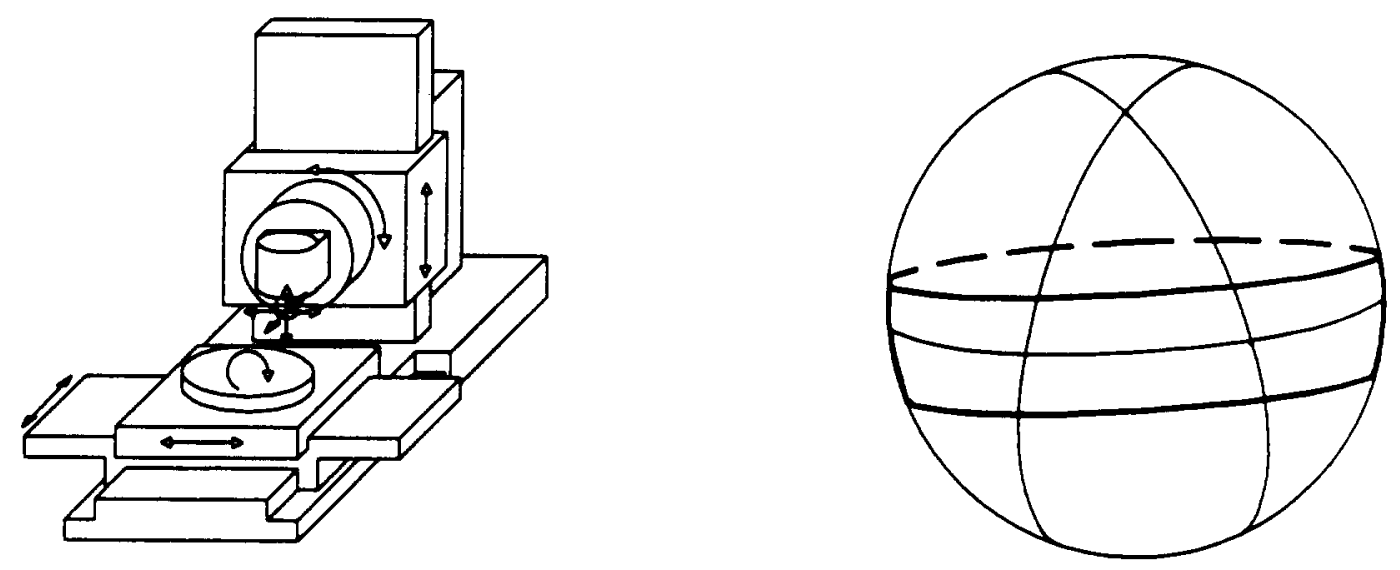

b

Figure 10 Machines; (a) 4-axis machine and great circle, (b) 5-axis machine and spherical band
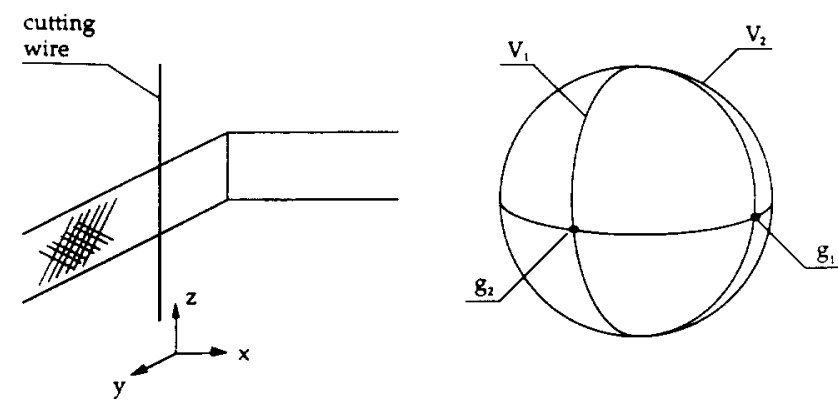

Figure 11 Wire-cutting EDM and line visibility

suffice, as the intersection of the $V \_$maps is a single point in this case. To accommodate more complex geometries of the workpiece, in wire-cutting EDM, the cutting wire needs additional degrees of freedom in rotation, giving rise to the 3-DOF and 4-DOF configurations in the middle of Figure 9, all of which have the generic form of the visibility problem.

The basic algorithms that enable the visibility problem to be solved are now easy to describe.

Intersection for constructing $V_{-}$maps: For point-visible processes, the $V \_$maps are constructed by intersecting hemispheres whose poles are points in the convex hull of the $G \_$maps of the workpiece surfaces. For line-visible processes, the $V_{-}$maps are obtained by intersecting great circles whose duals are points in the $G_{-}$maps. For surface-visible processes, the $V \_$maps are the $G_{-}$maps.

Intersection for orienting the workpiece: As in Figure 9, visibility is computed by intersecting a point, a great circle or a spherical rectangle with the $V_{-}$maps, depending on the degrees of freedom that are available in the mechanism of the manufacturing machine.

Since there can be many surfaces, and hence many $V \_$maps, it is desirable to intersect them optimally. A globally optimal solution to the problem of finding the minimum number of points (great circles or spherical rectangles) that intersect all the maps of interest is difficult. This has been proven ${ }^{12}$ to be equivalent to the set-covering problem, which is strongly NP-hard ${ }^{14}$. However, a locally optimal solution to the problem of finding one point (or a great circle or a spherical rectangle) that intersects the maximal number of maps is in hand. In particular, intersecting a point with the greatest number of maps is equivalent to finding the densest hemisphere. An $\mathrm{O}(n v \log n)$ time algorithm, where $n$ is the number of maps and $v$ is the total number of vertices in them, has recently emerged ${ }^{15}$. Now, 
intersecting a great circle with the greatest number of maps on the sphere is equivalent to sandwich cutting. It can also be achieved in $\mathrm{O}(n v \log n)$ time $^{15}$ by exploiting a novel partitioning scheme which reduces infinitely many possible solutions to a finite number of equivalent ones in $\mathrm{O}(n v)$ time $^{16}$.

\section{EXAMPLE}

The machining of an object with 12 surfaces (represented as planar, ruled, Coons and Bézier surfaces (the mixture was intentional)) on a 4-axis milling machine is shown ${ }^{17}$. The geometry of these surfaces is shown in Figure 12.

The $G \_m a p$ for the top surface (there are ten on the side and one at the bottom) is shown as the dark region on a unit sphere, which has been 'opened up' for clarity (see Figure 13). This surface will be referred to as 'surface number 12'. The $V_{-}$maps for all 12 surfaces are given in Figure 14, as incircle approximations to the convex V_maps.

These 12 (circular) maps are seen to overlap each other. Map 12, for example, overlaps map 2, which, in turn, overlaps maps 6 and 7. However, there is no overlap between maps 12 and 6 . A way of sorting out this intransitive relation is to use clustering. Table 1 shows, for example, that map $M_{2}$ is in cluster $x_{2}$ with map $M_{12}$. It is also in cluster $x_{1}$, with maps $M_{6}, M_{7}$ and $M_{3}$ (whose intersection is degenerated by the limiting view of Figure

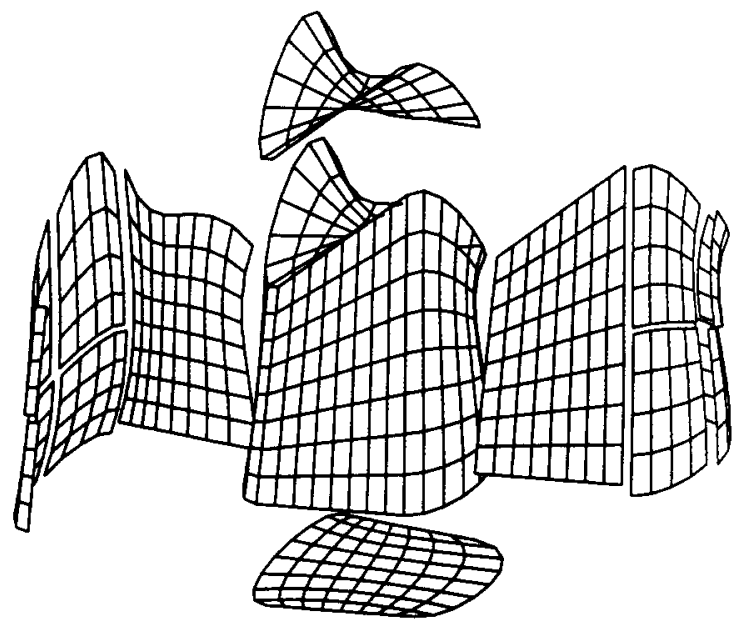

Figure 12 Object with 12 surfaces

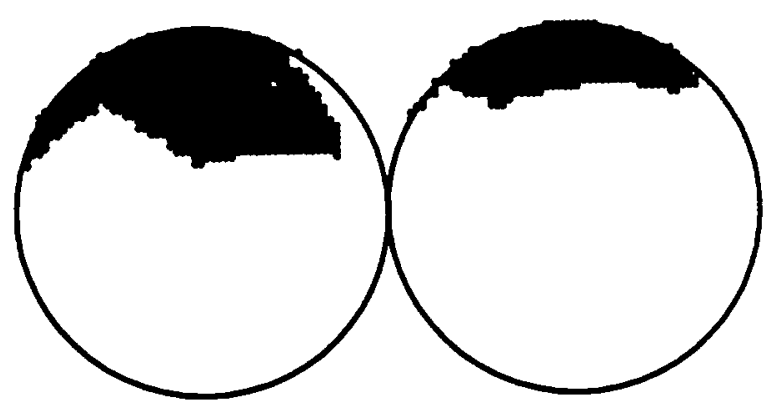

Figure 13 G_map for one surface

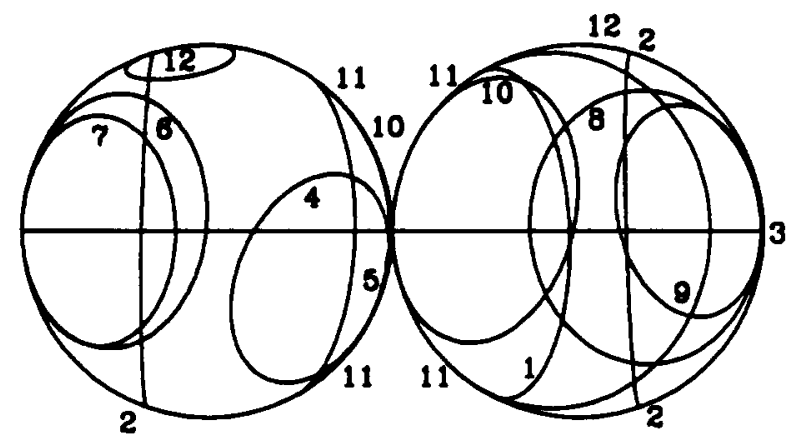

Figure $14 V \_$map for all 12 surfaces

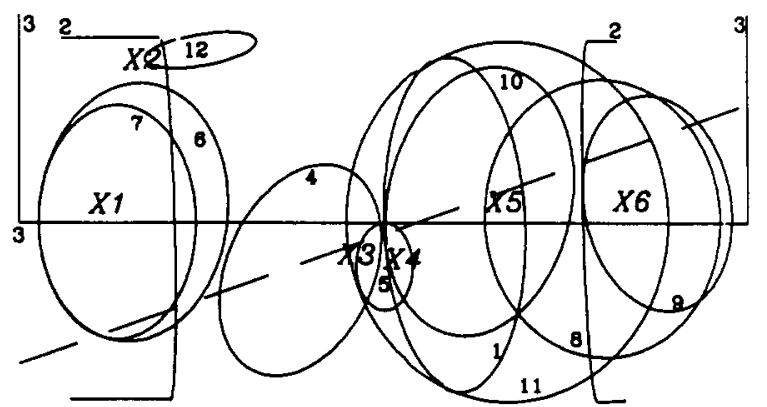

Figure 15 Intersecting the maps

Table 1 Clustering of maps

\begin{tabular}{lcccccc}
\hline Map & \multicolumn{5}{c}{ Cluster } \\
\hline & $x_{1}$ & $x_{2}$ & $x_{3}$ & $x_{4}$ & $x_{5}$ & $x_{6}$ \\
\hline$M_{1}$ & 1 & 1 & & 1 & 1 & \\
$M_{2}$ & 1 & & 1 & & 1 & 1 \\
$M_{3}$ & & & 1 & 1 & & \\
$M_{4}$ & 1 & & & & & \\
$M_{5}$ & 1 & & & & 1 & 1 \\
$M_{6}$ & & & & & 1 & 1 \\
$M_{7}$ & & & & 1 & 1 & 1 \\
$M_{8}$ & & & 1 & 1 & & \\
$M_{10}$ & & 1 & & & &
\end{tabular}

14. Table 1 reveals that map $M_{2}$ is also in cluster $x_{6}$, which contains a collection of maps, as shown on the right-hand side of Figure 14.

If a 3-axis machine is to be used, then five clusters $x_{1}$, $x_{2}, x_{3}, x_{4}$ and $x_{6}$ are needed, corresponding to five setups. If a 4-axis machine is to be used, only two setups are necessary. This is shown by the great circle shown by the broken line in Figure 15. This corresponds to the fourth axis, the rotating table. The broken line intersects all the maps, except for map $M_{12}$. Thus, the first setup on the 4-axis machine entails lining the cutter up (using three axes) with the great circle, and rotating the table (using the fourth axis). The second setup cleans up the remaining $M_{12}$. 


\section{CONCLUDING REMARKS}

The purpose of presenting visibility maps as data structures that support spherical algorithms is to enable toolpaths to be automatically generated, in a context that lies beyond the traditional NC machining of sculptured surfaces. Elsewhere, researchers have begun to analyse 'accessibility'18 for CMMs on the basis of the notion of a visibility 'cone'. Recently, 'global accessibility'19 has been considered to account for the partial visibility discussed in the third section.

In this paper, a wide range of manufacturing processes are categorized by their point, line and surface visibilities. Further, the various degrees of freedom in the mechanisms of the machines gives a computational structure in terms of the maximal intersection of a point, a great circle and a spherical rectangle with the maps.

The above formulation has been made possible by taking into account the scientific foundations laid down by researchers in three seemingly disparate areas: computer vision $^{20}$, geometric design ${ }^{5}$, and computational complexity ${ }^{21}$. Curiously, these three areas were all given the same name, computational geometry, a term coined independently by Minsky and Papert, Robin Forrest ${ }^{22}$ and Michael Shamos ${ }^{23}$. Perhaps this paper serves to unify these three connotations of computational geometry by offering a structure called visibility, from which efficient solutions to many classes of problem in design and manufacturing can now be systematically derived.

\section{ACKNOWLEDGEMENTS}

The author gratefully acknowledges the insight of the referees of this paper, and the financial support of the Fulbright Foundation, the US National Science Foundation (grants DDM-8903029 and DDM-9113791), and the University Research Fund of the Scientific Research Laboratory, Ford Motor Company, USA.

The work reported here would not have been possible if it were not for the diligence of the authors' colleagues at large: L L Chen (National Taiwan Institute of Technology, Taiwan), S Y Chou (National Taiwan Institute of Technology, Taiwan), J Gan (Nanyang Technological University, Singapore), S Y Shin (Korea Advanced Institute of Science and Technology, Korea), and $\mathrm{K}$ Tang (Schlumberger Technologies, USA) (listed in alphabetical order).

A preliminary version of this paper was given as an invited talk at the SIAM Conference on Geometric Design Tempe, AZ, USA (4-8 Nov 1991).

\section{REFERENCES}

1 O'Rourke, J Art Gallery Theorems and Algorithms Oxford University Press (1987)

2 Bézier, P Numerical Control: Mathematics and Applications John Wiley, UK (1972)
3 do Carmo, M P Differential Geometry of Curves and Surfaces Prentice-Hall (1976)

4 Hilbert, D and Cohn-Vossen, S Geometry and the Imagination Chelsea Publishing, USA (1983)

5 Faux, I D and Pratt, M J Computational Geometry for Design and Manufacture Halsted Press, USA (1979)

6 Farouki, R T 'The approximation of non-degenerate surfaces' Comput. Aided Geom. Des. Vol 2 No 1 (1985) pp 257-279

7 Chen, Y J and Ravani, B 'Offset surface generation and contouring in computer aided design' ASME Trans. J. Mechanisms, Transmission \& Automat. Des. Vol 109 (Mar 1987) pp 133-142

8 Sripradisvarakul, $\mathrm{T}$ and Jain, $\mathrm{R}$ 'Generating aspect graph for curved objects' Proc. IEEE Wkshp. Interpretation of $3 D$ Scenes Austin, TX, USA (27-29 Nov 1989) pp 109-115

9 Horn, B K 'Extended Gaussian image' Proc. IEEE Vol 72 No 12 (1984) pp 1671-1686

10 Chen, $\mathrm{L} \mathrm{L}$ and Woo, $\mathrm{T} \mathrm{C}$ 'Computational geometry on the sphere with application to automated machining' ASME Trans. J. Mech. Des. Vol 114 (Jun 1992) pp 288-295

11 Gan, J G, Woo, T C and Tang, $K$ 'Spherical maps: their construction, properties, and approximation' ASME Trans. $J$. Mech. Des. (to appear) (also available as Technical Report 91-3 Dep. Industrial \& Operations Engineering, University of Michigan, USA (Feb 1991))

12 Chen, L L 'Visibility algorithm for mold and die design' $P h D$ Dissertation Dep. Industrial \& Operations Engineering, University of Michigan, USA (1991)

13 Tang, $\mathrm{K}$ and Woo, $\mathrm{T} \mathrm{C}$ 'Algorithmic aspects of alternating sum of volumes. Part I: Data structure and difference operation' Comput.-Aided Des. Vol 23 No 5 (1991) pp 357-366

14 Megiddo, $\mathrm{N}$ and Tamir, $\mathrm{A}$ 'On the complexity of locating linear facilities in the plane' Oper. Res. Lett. Vol 1 No 5 (1982) pp 194-197

15 Chen, L L, Chou, S Y and Woo, T C 'Separating and intersecting spherical polygons for computing visibility on 3-, 4-, and 5-axis machines' ACM Trans. Graph. (to appear) (also available as Technical Report 91-9 Dep. Industrial \& Operations Engineering, University of Michigan, USA (Mar 1991))

16 Tang, K, Woo, T C and Gan, J Maximum intersection of spherical polygons and workpiece orientation for 4- and 5-axis machining' ASME Trans. J. Mech. Des. Vol 114 (Aug 1992) pp $271-276$

17 Gan, J G 'Spherical algorithms for setup orientations for workpieces with sculptured surfaces' $P h D$ Dissertation Dep. Industrial \& Operations Engineering, University of Michigan, USA (1990)

18 Spyridi, A J and Requicha, A A G 'Accessibility analysis for the automatic inspection of parts' Proc. IEEE Int. Conf. Robotics \& Automation Cincinnati, OH, USA (13-18 May 1990) pp 1284-1289

19 Requicha, A A G 'Computing global accessibility directions for the faces of a solid' SIAM Conf. Geometric Design Tempe, AZ, USA (4-8 Nov 1991)

20 Minsky, M and Papert, S Perceptions: An Introduction to Computational Geometry MIT Press (1972)

21 Preparata, F P and Shamos, M I Computational Geometry - An Introduction Springer-Verlag (1985)

22 Forrest, A R 'Computational geometry' Proc. Roy. Soc. Vol 321 Ser. 4 (1971) pp 187-195

23 Shamos, M I 'Computational geometry' PhD Dissertation Dep. Computer Science, Yale University, USA (1978)

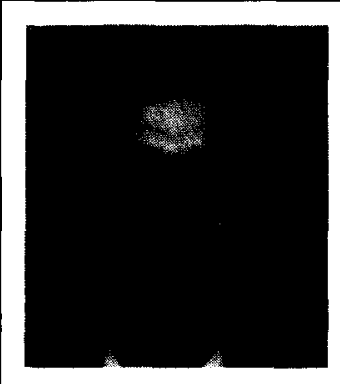

Tony $C$ Woo is a professor of industrial and operations engineering and $a$ professor of mechanical engineering and applied mechanics. His research interest is in the development of efficient data structures and algorithms for design and manufacturing. He has been a Fulbright Scholar at the NTH, Trondheim, Norway, and has served at the US National Science Foundation, Washington DC, USA. 